\title{
ARQUITECTURA DISTRIBUIDA PARA EL CONTROL AUTÓNOMO DE DRONES EN INTERIOR
}

\author{
Jose-Luis Poza-Lujan \\ Camino de vera, s/n. 46022 Valencia, jopolu@ai2.upv.es \\ Juan-Luis Posadas-Yagüe \\ Camino de vera, s/n. 46022 Valencia, jposadas@ai2.upv.es \\ Giovanny-Javier Tipantuña-Topanta \\ Camino de vera, s/n.46022 Valencia, giotitoa@upvnet.upv.es \\ Francisco Abad \\ Camino de vera, s/n. 46022 Valencia, fjabad@ai2.upv.es \\ Ramón Mollá \\ Camino de vera, s/n. 46022 Valencia, rmolla@ai2.upv.es
}

\section{Resumen}

Actualmente los drones son uno de los sistemas de control más complejos. Este control va desde el control de la estabilidad del propio dron, hasta el control automático de la navegación de dicho dron en entornos complejos. En el caso de drones que deben navegar en interior los retos tecnológicos son especificos. En el presente artículo se muestra la arquitectura de control inteligente de un dron orientado a la navegación en entornos interiores. La seguridad es el eje principal del diseño del sistema. Esto hace que el principal reto de la arquitectura sea la interconexión segura entre los componentes y la definición de los diferentes métodos de navegación basándose en la seguridad. El dron debe disponer de diversos modos de navegación: manual, reactivo, deliberativo e inteligente. Para la navegación en interior es necesario conocer la posición del dron en todo momento, por ello el sistema debe disponer de un modo de localización similar al GPS, pero que proporcione una precisión mucho mayor. Para los modos deliberativo e inteligente, el sistema debe disponer de un mapa del entorno, así como de un sistema de control que envie al dron las órdenes de navegación correspondientes. El sistema diseñado se está implementando en el marco del proyecto europeo H2020 AiRT (Arts indoor RPAS Technology transfer). El resultado es una propuesta de arquitectura suficientemente versátil para ser empleadas en sistemas similares y servir como base de diseño para futuras implementaciones.

Palabras Clave: Dron; Navegación en interiores; Sistema distribuido.

\section{INTRODUCCION}

Los drones son ya una realidad tecnológica que se ha instaurado tanto en la vida cotidiana como en los entornos de trabajo. Los entornos de actuación de los drones pueden ser interiores y exteriores. La mayor parte de los drones tienen un control autónomo para proporcionar la estabilidad y dar soporte a los movimientos del mismo. En el caso de que el dron deba realizar misiones, se precisa de un control autónomo. El control autónomo implica un seguimiento de una ruta, y para dicho seguimiento es necesario disponer de un mapa del entorno, de un mecanismo de posicionamiento dentro de dicho mapa y de un sistema de navegación. Además del modo de pilotaje manual, el sistema de navegación debe disponer, a su vez, de diversas capas. Una capa reactiva que se ocupe de la integridad tanto del dron como del entorno, una capa deliberativa que permita al dron navegar en un entorno conocido, principalmente partiendo del uso del mapa, y una capa inteligente que permita al dron seguir una ruta predefinida. Para poder seguir una ruta en un entorno, el dron necesita de un sistema de localización. En el caso de drones que trabajan en entornos exteriores la localización la proporciona el sistema GPS. Sin embargo, en el caso de los drones que deben navegar en entornos interiores, la localización debe tener mucha más precisión que en entornos exteriores.

Tanto en entornos exteriores como en interiores, la seguridad es el aspecto más importante. En el caso de drones de navegación en entornos exteriores, lo más habitual es que el entorno de trabajo de dichos drones sean entornos poco poblados, dado que su vuelo en núcleos poblacionales está muy limitado. 
Sin embargo, los entornos interiores suelen ser entornos donde es habitual encontrar personas, pero también elementos de valor. Por ello, la seguridad que debe proporcionar un dron en interior debe ser mayor. Este aspecto determina, por lo tanto, en gran medida todas las cuestiones relativas al diseño, tanto de la arquitectura de control como del dron.

Para determinar claramente estos aspectos surge el proyecto europeo AiRT (Arts indoor RPAS Technology transfer) en el que se estudia cómo integrar la navegación autónoma en tiempo real de drones en espacios interiores. La finalidad del uso de drones en espacios interiores es emplearlos para la industria creativa. Esta industria precisa del uso de cámaras de muy alta definición y de un soporte o gimbal (cardán) que proporcione a la cámara los suficientes grados de libertad como para realizar las grabaciones necesarias.

Los aspectos previos se presentan en el artículo y se organizan de la siguiente forma: inicialmente se presenta la problemática que trata de resolver el proyecto AiRT. En el siguiente apartado se estudian las necesidades del sistema, especialmente los relativos a la seguridad del entorno y del dron. Seguidamente se presentan los elementos de la arquitectura que dan soporte al sistema para finalizar con la discusión de la experiencia obtenida en los primeros meses de proyecto.

\section{EL USO DE DRONES EN ENTORNOS INTERIORES}

En el entorno de la industria creativa, para dotar de la mayor cantidad de movimientos posibles a la cámara, es habitual el uso de una gran cantidad de dispositivos como grúas, raíles o armazones portátiles. Estos dispositivos suelen tener muchos inconvenientes: son complejos de montar, manejar y desmontar, aunque la mayor limitación es que tienen un espacio limitado de movimientos. Sin embargo, los vehículos aéreos no tripulados, conocidos como UAVs, RPAs o drones, evitan los inconvenientes anteriormente nombrados. Cuando la grabación se produce en entornos interiores, desde un plató de televisión o cine hasta pabellones deportivos cubiertos, los drones pueden proporcionar tomas desde puntos de vista imposibles con los medios actuales dado que su estabilidad es controlable con una gran precisión [2].

Sin embargo, un dron que realice grabaciones de entornos interiores, requiere de dos características fundamentales: seguridad de todos los elementos (dron y entorno, especialmente si se encuentran personas) y control en tiempo real del dron y de la navegación del mismo. Actualmente la navegación en interior de drones se realiza mayoritariamente usando drones comerciales, tanto para el control del dron [4] como para el seguimiento de la trayectoria [9]. Siendo éste último aspecto uno de los más interesantes en cuanto a investigación se refiere [6].

Los aspectos de navegación y control en tiempo real del dron son especialmente relevantes para la industria creativa. La posibilidad de disponer de la trayectoria realizada por el dron permite repetir las grabaciones (algo muy habitual en la industria creativa). Por ello, es necesario un sistema de soporte que proporcione las rutas realizadas, o permita diseñar rutas para el dron dentro del entorno. Este sistema, además, implica disponer de un mapa en tres dimensiones del entorno interior, siendo los drones los vehículos más favorables para ser empleados con este fin [3].

\section{ARQUITECTURA: SEGURIDAD Y CONTROL}

\subsection{EL ENTORNO}

El entorno de navegación en interior para un dron se caracteriza por tener en cuenta completamente las tres dimensiones. En entornos robóticos, generalmente son dos dimensiones, junto al área de actuación vertical del robot, las que se tienen en cuenta. Sin embargo, los drones tienen una libertad de movimiento similar en todas las dimensiones. Consecuentemente, cada dimensión tiene una serie de limitaciones que se deberán considerar. La figura 1 , muestra los límites en el caso menos favorable (el eje vertical).

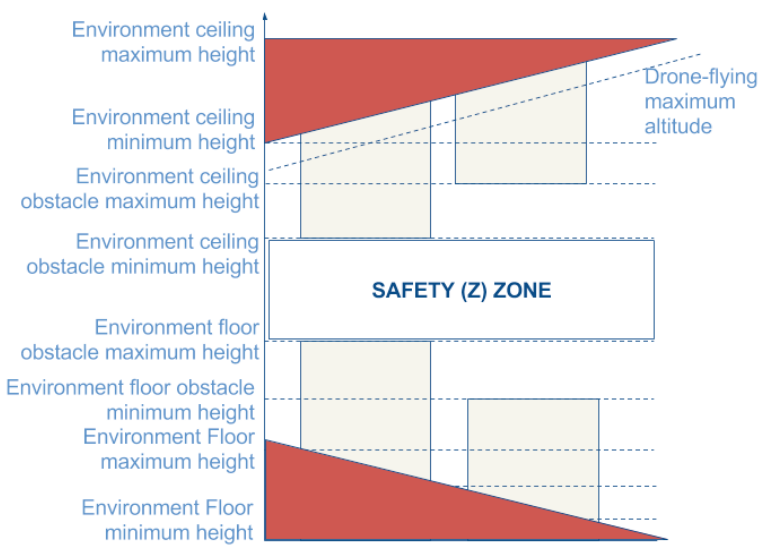

Figura 1: Límites de actuación del dron en el entorno (caso concreto del eje z).

En el centro de la figura 1 se destaca la zona segura en la que sólo se pueden encontrar obstáculos no predecibles, generalmente en movimiento. Los condicionantes del eje vertical son extrapolables al 
resto de los ejes. Por ello el mapa del entorno sólo cubre parte de la seguridad, y es el propio dron el que deberá disponer de los mecanismos necesarios para poder detectar aquellos obstáculos no incluidos en el mapa. El hecho de disponer de un mapa, permite determinar las zonas donde, bien por encontrarse obstáculos conocidos o bien por otros motivos, no se desea que el dron vuele.

\subsection{NIVELES DE SEGURIDAD}

La navegación del dron en un entorno interior con las características descritas en el primer apartado debe tener diversos niveles de seguridad. Dichos niveles de seguridad están relacionados con los niveles de navegación que tiene el dron (figura 2)

\begin{tabular}{|c|c|c|c|c|}
\hline & $\begin{array}{c}\text { Manual } \\
\text { navigation }\end{array}$ & $\begin{array}{c}\text { Reactive } \\
\text { navigation }\end{array}$ & $\begin{array}{l}\text { Deliberative } \\
\text { navigation }\end{array}$ & $\begin{array}{l}\text { Intelligent } \\
\text { navigation }\end{array}$ \\
\hline $\begin{array}{l}\text { Avoiding } \\
\text { obstacles }\end{array}$ & No & Yes & Yes & Yes \\
\hline $\begin{array}{c}\text { Avoiding } \\
\text { forbidden places }\end{array}$ & No & No & Yes & Yes \\
\hline $\begin{array}{c}\text { Follow the flight } \\
\text { path }\end{array}$ & No & No & No & Yes \\
\hline
\end{tabular}

Figura 2: Niveles de navegación y características relacionadas.

El principal nivel de seguridad es el que contempla que, incluso, los sensores que proporcionan la detección y evitación de obstáculos al dron pueden fallar, es por ello que el dron debe, siempre, poder ser pilotado de forma manual. El siguiente nivel de seguridad es el nivel reactivo, donde los sensores incorporados al dron deben detectar los obstáculos y el sistema reactivo evitarlo. El nivel deliberativo es el que permite al dron volar en los espacios que, en el mapa, están autorizados, comprobar si se sigue una ruta o, incluso, generar rutas. Esto se debe a que la navegación deliberativa implica el uso del mapa para aquellos aspectos en que pueda ser de utilidad. Finalmente, el nivel inteligente es aquel en el que la navegación no es pilotada por personas, sino que el sistema es el que guía al dron. Para este guiado, además de disponer de un mapa, se debe disponer de una ruta ya establecida que el dron deberá seguir. Los niveles de seguridad son prioritarios. La navegación manual tiene prioridad sobre todos los modos. La reactiva, es prioritaria sobre la deliberativa y la inteligente, siendo esta última la de menor prioridad.

\section{ARQUITECTURA PROPUESTA}

A partir de las características anteriores, se diseñan los componentes de la arquitectura del sistema. Dado que debe haber una navegación deliberativa $\mathrm{e}$ inteligente, el sistema debe disponer de un mapa y de un método de posicionar al dron dentro de dicho mapa. Además, la navegación inteligente precisa de un método de especificación de rutas a seguir y de acciones a tomar durante la navegación en dicha ruta. Las acciones son las propias de la misión del dron, en concreto son acciones de navegación (movimiento entre puntos, instante temporal y velocidad a la que debe moverse) y acciones de grabación (parámetros de la cámara de grabación, orientación de cámara, instantes en que debe empezarse y debe finalizar la grabación, etc. En la figura 3, se muestran los principales bloques del sistema.
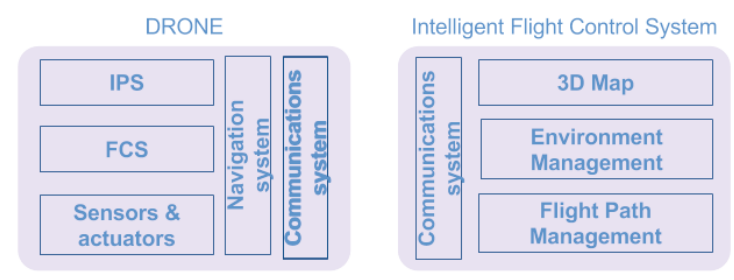

Figura 3: Bloques de la arquitectura propuesta.

\subsection{DRON}

El dron empleado es un diseño de la empresa Aerotools UAV [1]. El dron dispone de una serie de sensores y actuadores propios de este tipo de vehículos, tales como el Global Positioning System (GPS) o el Inertial Measurement Unit (IMU). Sin embargo, para la navegación reactiva, son necesarios más sensores, en concreto un sistema de sensores de rango, que permiten detectar y evitar obstáculos, y un Indoor Positioning System (IPS) que suple al GPS en entornos interiores. En el caso de los actuadores, se dispone de los actuadores propios de un dron (motores y balizas), pero incrementados con zumbadores que permitan avisar acústicamente en el caso de situaciones de emergencia. La lectura de los sensores, y actuación correspondiente, es realizada por el Flight Control System (FCS).

Para la creación del mapa, el dron va equipado con una cámara RGBD ampliamente usada para tareas similares de reconstrucción 3D [7]. Para la grabación, el dron dispone de un cardán que debe ser controlable y una cámara de grabación $4 \mathrm{~K}$. Las cámaras para el mapa y para la grabación son gestionadas por el sistema de navegación.

\subsection{SISTEMA DE POSICIONAMIENTO INTELIGENTE (IPS)}

El sistema de posicionamiento inteligente se basa en tecnología Ultra Wide Band (UWB) [5]. Este sistema consiste en una serie de antenas situadas en el entorno y un receptor situado en el dron. El sistema está desarrollado por la empresa Pozyx [8]. 


\subsection{SISTEMA INTELIGENTE NAVEGACION AUTÓNOMA}

Las tareas principales de este módulo son la creación del mapa, la asistencia al usuario para la creación del plan de vuelo y la monitorización de los vuelos.

Para la creación del mapa 3D del espacio donde se moverá el dron se utilizará una cámara de profundidad a bordo. Dicha cámara es capaz de capturar tanto la geometría de la escena como su textura. El resultado de una captura de dicha cámara es una nube de puntos (figura 4), donde cada punto está definido por sus 3 coordenadas $(\mathrm{X}, \mathrm{Y}, \mathrm{Z})$ con respecto a la cámara, y su color. Para construir un mapa global de la escena, es necesario transformar cada una de las nubes de puntos capturadas a un sistema de coordenadas común. El sistema de posicionamiento proporciona en cada momento la posición y orientación del dron (y, por lo tanto, de la cámara), lo que permite llevar cada punto del sistema de la cámara al sistema de coordenadas común. De esta forma el alineamiento de varias nubes de puntos resultará en un modelo $3 \mathrm{D}$ consistente con mínimas distorsiones (las debidas a la precisión de los sensores empleados). Para optimizar este proceso es necesario eliminar aquellos puntos que se corresponden con el ruido inherente del sensor de profundidad y las condiciones de iluminación. Así mismo, durante el alineamiento de las nubes de puntos, se deben descartar aquellos puntos que se encuentran en una zona que ya ha sido registrada en el modelo 3D global y añadir únicamente aquellos que se encuentran en zonas no registradas.

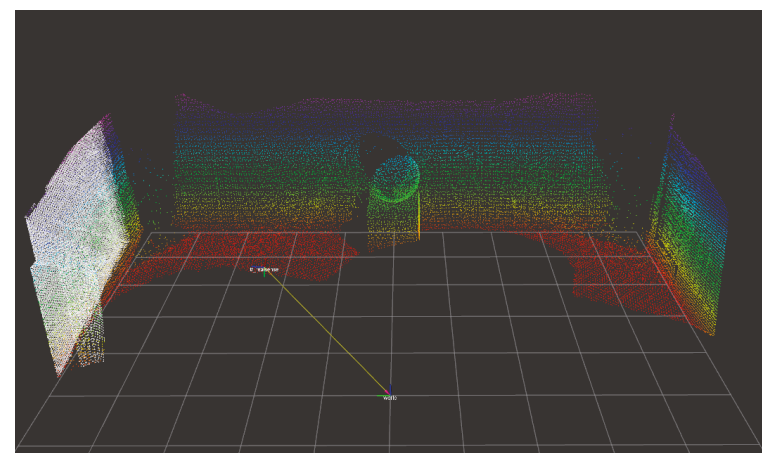

Figura 4: Simulación del alineamiento de nubes de puntos.

Una vez que se ha capturado el mapa del entorno, el sistema permitirá al usuario definir el plan de vuelo. El plan de vuelo consiste, como se ha explicado antes, en una secuencia de puntos de paso (waypoints) por los que tiene que pasar el dron, y la configuración del gimbal y la cámara. Para realizar dicho plan de vuelo, el sistema mostrará al usuario una interfaz de usuario con varias vistas del mapa capturado en el paso anterior. El usuario podrá definir el camino que debe seguir el dron, y la posición y orientación de la cámara en cada punto del camino. Para ello tan sólo deberá manejar una cámara virtual, que le mostrará una aproximación de lo que verá la cámara real, una vez que el dron ejecute el plan de vuelo, y se encuentre en dicho punto. Definir el plan de vuelo off-line permite no depender de la duración de las baterías del dron y poder trabajar en cualquier momento y lugar, incluso sin acceso al dron. Por último, la interfaz de usuario proporcionará herramientas para ajustar el plan de vuelo durante la realización del vuelo. El usuario tendrá varias opciones para controlar el plan de vuelo. Por ejemplo, el dron puede actuar como una cable cam. Este dispositivo consiste en un cable instalado en la zona de grabación, del que cuelga la cámara. La cámara está instalada sobre una plataforma motorizada, que permite avanzar o retroceder sobre el cable. Siguiendo esta metáfora, se podría considerar que el plan de vuelo es el cable (es mucho más flexible, puesto que puede seguir formas más complejas que un cable real), y entonces el operador podría, durante la grabación, tener herramientas para avanzar, retroceder, parar, acelerar o decelerar a lo largo de ese cable virtual. Esta interfaz permite a un usuario poco experimentando en el vuelo de drones realizar vuelos complejos fácilmente.

\subsection{COMUNICACIONES}

El sistema de comunicaciones es el responsable de gestionar la conexión entre todos los elementos. Esta conexión se da entre los propios elementos del dron y entre el dron y el sistema de navegación inteligente.

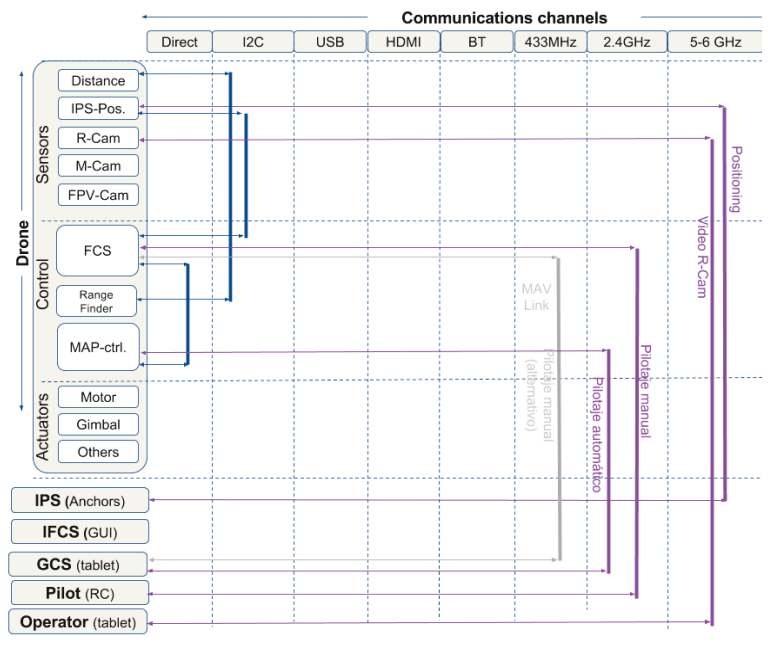

Figura 5: Interconexión de los componentes del sistema.

El principal reto de las comunicaciones está en la heterogeneidad de los componentes y en los requisitos que los diferentes modos de navegación imponen. En la figura 5 se muestran los componentes y las conexiones entre ellos. 
Internamente el dron se conecta por medio de enlaces directos y el bus I2C. Dejando la conexión USB y HDMI como medios auxiliares de soporte en caso de necesitarse una vía alternativa. La conexión entre el dron y el sistema de control (tanto el piloto como el sistema automático inteligente) se realiza por medio de diferentes canales. El modo de navegación manual emplea la frecuencia de $2.4 \mathrm{GHz}$, dado que esta frecuencia también la emplea el control automático, simplificándose de esta forma el acceso a las órdenes por parte del dron. Sin embargo, el modo de navegación manual también puede emplear la frecuencia de $433 \mathrm{MHz}$ como modo de emergencia. Ya que la navegación manual es la considerada de emergencia debe poder facilitarse dos canales para dar soporte al fallo de uno de ellos.

El sistema de posicionamiento emplea la frecuencia de $5 \mathrm{GHz}$. Esta frecuencia es compartida, por medio de bandas, con la emisión de la cámara de grabación. Esto se debe a que la cámara de muy alta definición precisa de un considerable ancho de banda para poder enviar las imágenes de vídeo correspondientes.

\section{FUNCIONAMIENTO}

Los componentes presentados anteriormente se ponen en marcha en diferentes fases, numeradas en función del vuelo del dron que se responsabiliza de la misma. Las fases se muestran en la figura 6.

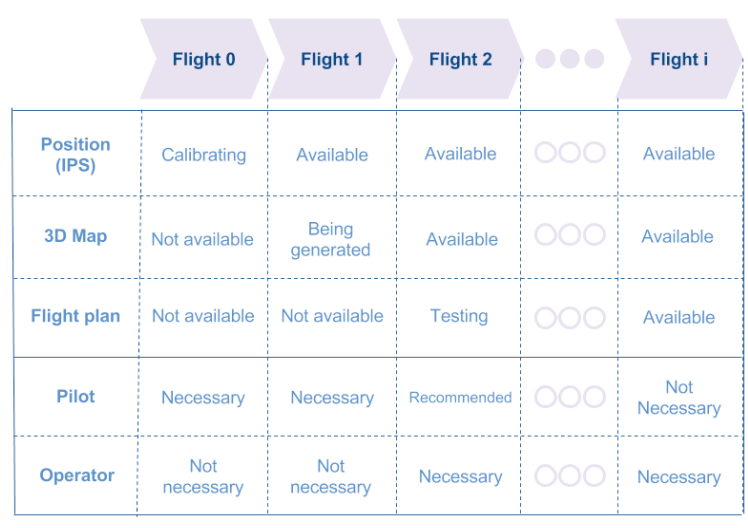

Figura 6: Funcionamiento del sistema propuesto.

La fase inicial (vuelo 0) es la que se emplea para calibrar el sistema de posicionamiento, dado que la posición del dron es fundamental para la creación del mapa. Una vez se ha calibrado el sistema de posicionamiento, se pasad al vuelo 1 , donde debe construirse el mapa usando la cámara RGBD. Dado que en estas dos etapas no se dispone de mapa, el dron debe ser pilotado por personal experto. El vuelo 2 , es el primero en el que se dispone de la posición del dron y del mapa del entorno. En este vuelo no es imprescindible contar con un piloto, dado que el objetivo principal del mismo es determinar el plan de vuelo (incluyendo las acciones que debe realizar la cámara de grabación).

Cada uno de los diferentes vuelos de la puesta en funcionamiento se asocian con modos de navegación. El vuelo 0 y el vuelo 1 se asocian al modo de navegación reactivo. Una vez creado el mapa se puede realizar el vuelo 2, asociado al modo de navegación deliberativo. De esta forma, conociendo el entorno y las zonas de vuelo permitidas, el operador ya puede decidir qué tomas desea realizar. El vuelo 2 puede repetirse las veces que sea necesario para así dejar el plan de vuelo listo para los posteriores vuelos, que ya pueden realizarse en modo de navegación inteligente.

\section{CONCLUSIONES}

En el presente artículo se ha mostrado la arquitectura inicial del proyecto AiRT. Esta arquitectura está orientada a lograr la navegación autónoma de un dron en entornos interiores. La misión del dron es la grabación de vídeos y fotografías con cámaras de muy alta definición. En el artículo se ha mostrado las dificultades que este tipo de misión implican. Para lograr realizar este tipo de misiones, el sistema precisas de diferentes modos de navegación. Lo cual determina proporcionar al dron de una cantidad de sensores no habitual y de un sistema de posicionamiento en interiores.

Actualmente se tienen dos retos concretos para alcanzar como metas del proyecto. El primero de ellos consiste en lograr parametrizar correctamente la medición de la navegación del dron, ya que no sólo deben tenerse en cuenta los parámetros clásicos de la navegación de robots en entornos interiores, sino también los cambios entre modos de navegación. El hecho de que los entornos interiores se compongan de volúmenes, por lo general, más pequeños que los entornos exteriores hace que los cambios entre modos deban realizarse en tiempo real estricto. El siguiente de los retos consiste en lograr una navegación autónoma completa. Esto implica que el dron no deba depender en ningún momento de pilotaje experto. Este aspecto implica que los vuelos de calibración y aprendizaje del entorno deban realizarse de forma autónoma sin un mapa y sin un sistema de posicionamiento calibrado. Este reto incluye el uso de técnicas de SLAM y de aprendizaje automático.

Finalmente, el proyecto tiene dos posibles ramas para continuar desarrollándose: la navegación híbrida entre entornos interiores y exteriores, y la navegación simultánea de varios drones compartiendo mapa y sistema de posicionamiento. 


\section{Agradecimientos}

Este proyecto está financiado por el programa de la Unión Europea Horizonte 2020 de código No 732433 - AiRT.

\section{Referencias}

[1] AeroTools-UAV. [Online: julio 2017]. Available: www.aerotools-school.es

[2] Castillo, P., García, P., Lozano, R., \& Albertos, P. (2007). Modelado y estabilización de un helicóptero con cuatro rotores. Revista Iberoamericana de Automática e Informática Industrial RIAI, 4(1), 41-57.

[3] Heng, L., Honegger, D., Lee, G. H., Meier, L., Tanskanen, P., Fraundorfer, F., \& Pollefeys, M. (2014). Autonomous visual mapping and exploration with a micro aerial vehicle. Journal of Field Robotics, 31(4), 654-675.

[4] Hussein, A., Al-Kaff, A., de la Escalera, A., \& Armingol, J. M. (2015, November). Autonomous indoor navigation of low-cost quadcopters. In Service Operations And Logistics, And Informatics (SOLI), 2015 IEEE International Conference on (pp. 133-138). IEEE.

[5] Lee, J. S., Su, Y. W., \& Shen, C. C. (2007, November). A comparative study of wireless protocols: Bluetooth, UWB, ZigBee, and Wi-Fi. In Industrial Electronics Society, 2007. IECON 2007. 33rd Annual Conference of the IEEE (pp. 46-51). IEEE.

[6] Martinez, S. E., \& Tomas-Rodriguez, M. (2014). Three-dimensional trajectory tracking of a quadrotor through PVA control. Revista Iberoamericana de Automática e Informática Industrial RIAI, 11(1), 54-67.

[7] Munera, E., Poza-Lujan, J. L., Posadas-Yagüe, J. L., Simó-Ten, J. E., \& Noguera, J. F. B. (2015). Dynamic reconfiguration of a RGBD sensor based on QoS and QoC requirements in distributed systems. Sensors, 15(8), 1808018101.

[8] Pozyx - Accurate Positioning. [Online: julio 2017]. Available: http://www.pozyx.io
[9] Santana, L. V., Brandao, A. S., Sarcinelli-Filho, M., \& Carelli, R. (2014, May). A trajectory tracking and $3 \mathrm{~d}$ positioning controller for the ar. drone quadrotor. In Unmanned Aircraft Systems (ICUAS), 2014 International Conference on (pp. 756-767). IEEE. 